\title{
Are household disinfectants microbially mediated obesogens?
}

\author{
Moira K. Differding BA, Noel T. Mueller MPH PhD
}

— Cite as: CMAJ 2018 September 17;190:E1095-6. doi: 10.1503/cmaj.181134

See related article at www.cmaj.ca/lookup/doi/10.1503/cmaj.170809

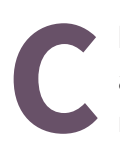

hildhood obesity continues to rise in prevalence globally, and is associated with lifelong morbidity and premature mortality. Because losing weight and keeping it off later in life have proven difficult, ${ }^{1}$ the best chance to curb the global rise in obesity is through prevention early in life. ${ }^{2}$ Yet, to do so, we must continue to identify modifiable risk factors in early life associated with childhood obesity. In linked research, Tun and colleagues consider how early-life exposure to household cleaning agents is associated with features of the infant gut microbiome and childhood body mass index (BMI), to explore whether early postnatal exposure to household disinfectants should be added to the list of possible infant-microbiome disruptors that increase risk of childhood obesity. ${ }^{3}$

Exposure to commensal microbes during infancy is at once important for both education of the immune system ${ }^{4}$ and programming of the metabolic system. ${ }^{5}$ In prospective birth cohort studies, disrupting natural development of the infant gut microbiome via cesarean delivery, ${ }^{6}$ prenatal $^{6}$ and postnatal ${ }^{7}$ antibiotics, and formula feeding ${ }^{8}$ has been associated with higher risk of childhood obesity. Disinfectants may be pernicious to the microbiome and metabolism in a similar regard. Frequent use of household disinfectants reduces the presence of microbes in the home. ${ }^{9}$ Piglets reared in a disinfectant-treated environment showed signs of a compromised microbiome. ${ }^{10}$ Moreover, some household disinfectants contain antibacterial endocrinedisrupting agents (e.g., triclosan) ${ }^{11}$ that have been associated with altered growth of children. ${ }^{12}$

In light of this literature, Tun and colleagues examined associations of household cleaning agents, the infant gut microbiome (assessed through fecal samples collected at three to four months of age) and childhood BMI $z$ scores at age one and three years. ${ }^{3}$ They used survey data to estimate the use of household disinfectants, which typically contain higher concentrations of antimicrobial chemicals that can adversely affect the microbiome, and ecofriendly products, which might be beneficial or less harmful alternatives. ${ }^{11,12}$ They report associations of use of household disinfectants and eco-friendly products with several outcomes: overall composition of the bacterial community, differential abundance of specific bacterial taxa, and one- and three-year BMI z score. ${ }^{12}$

\section{KEY POINTS}

- The prevalence of childhood obesity continues to rise globally, making it more important than ever to identify modifiable risk factors for prevention.

- High use of disinfectants has been shown to reduce household presence of microbes and to alter the microbiome in piglets; endocrine-disrupting agents present in many products are capable of negatively affecting child growth.

- New research has examined associations between household cleaning agents, the microbiome at three to four months and childhood obesity, finding an association between high disinfectant use and obesity that may be partly mediated by Lachnospiraceae.

- Although these associations are biologically plausible, further research is needed to understand whether residual confounding might have accounted for current findings, and to provide stronger evidence on whether endocrine-disrupting agents in antimicrobial cleaners are obesogenic.

Specifically, frequent use of household disinfectants (defined as $>30$ th percentile) was associated with higher child BMI $z$ score, whereas above-median use of eco-friendly products was associated with lower child BMI $z$ score. ${ }^{3}$ Higher disinfectant use was also positively associated with the family Lachnospiraceae but inversely associated with the genus Haemophilus, while households with higher use of eco-friendly products had lower abundance of the family Enterobacteriaceae. ${ }^{3}$ The doseresponse trends for household cleaning agents with Lachnospiraceae and Enterobacteriaceae, along with their associations with child BMI, made these particular taxa eligible for mediation analyses. In these analyses, Lachnospiraceae mediated part of the association between high use of disinfectants and child BMI, whereas Enterobacteriaceae did not play a mediating role in associations with use of eco-friendly products.

There is biologic plausibility to the finding that early-life exposure to disinfectants may increase risk of childhood obesity through the alterations in bacteria within the Lachnospiraceae family. An experimental study showed that piglets raised in a disinfectant-treated environment have higher abundance of 
Lachnospiraceae. ${ }^{10}$ Other than the Canadian Healthy Infant Longitudinal Development (CHILD) study, ${ }^{8}$ birth cohort studies examining abundance of Lachnospiraceae in the infant microbiome in relation to childhood BMI are sparse and often conducted at varying developmental stages, making comparisons difficult. ${ }^{14}$ Strains of Lachnospiraceae found in the human gut microbiome can be proficient short-chain fatty acid producers and even potentially influence immune cell signalling, giving them the means to participate in obesity-associated pathways. ${ }^{4,13}$ However, because the functions of these strains may vary markedly, more granular examination at the species or strain level is needed, to gain inference into mechanistic pathways.

Although the findings of the current study are intriguing, and strengthened by a sample size larger than previous birth cohorts with microbiome data, there are also several limitations. The crude odds ratio for high disinfectant use and child BMI $z$ score was only borderline significant, and only when a 30th percentile cut-off for disinfectants was used; it was not significant for cut-offs at the 40th or 50th percentile. ${ }^{3}$ It is unclear whether this association would have remained significant in a multivariable model excluding Lachnospiraceae. The association of household disinfectants with Lachnospiraceae was also only borderline significant, after decreasing markedly from the crude to the multivariable adjusted model. ${ }^{3}$ The influence of residual confounding on the reported associations cannot be ruled out. Furthermore, the authors did not have direct measurement of the endocrine-disrupting agents (e.g., triclosan) that motivated this investigation in the first place.

More research is needed to explore the intriguing possibility that use of household disinfectants might contribute to the complex causes of obesity through microbially mediated mechanisms. Future longitudinal birth cohort studies will need to incorporate serial measurements of household cleaning agents, the infant microbiome and childhood adiposity to confirm the findings of the current study. Moreover, future studies on this topic would be strengthened by consistently adjusting for confounders identified in the linked study (e.g., maternal pre-pregnancy BMI, maternal education, maternal smoking and geography), as well as from measuring and including factors not considered by Tun and colleagues, including household income, the timing of solid food introduction and child diet. If the underlying biologic mechanism between disinfectants and obesity involves triclosan or a combination of endocrine-disrupting chemicals, quantifying these exposures with urine or blood samples would provide mechanistic insight that could have important policy implications. Finally, although $16 \mathrm{~S}$ amplicon sequencing data are useful for identifying initial associations, future research that makes use of shotgun metagenomic sequencing would help provide more granular taxonomic resolution and elucidate microbial functionality, which could be leveraged to formulate probiotics that could restore optimal gut microbiota in affected infants.

\section{References}

1. Fothergill E, Guo J, Howard L, et al. Persistent metabolic adaptation 6 years after “The Biggest Loser” competition. Obesity (Silver Spring) 2016;24:1612-9.

2. Belsky DW. Appetite for prevention: genetics and developmental epidemiology join forces in obesity research. JAMA Pediatr 2014;168:309-11.

3. Tun MH, Tun HM, Mahoney JJ, et al. Postnatal exposure to household disinfectants, infant gut microbiota and subsequent risk of overweight children. CMAJ 2018;190:E1097-107.

4. Ximenez C, Torres J. Development of microbiota in infants and its role in maturation of gut mucosa and immune system. Arch Med Res 2017;48:666-80.

5. Cox, L. M. et al. Altering the intestinal microbiota during a critical developmental window has lasting metabolic consequences. Cell 2014;158:705-21.

6. Mueller NT, Whyatt R, Hoepner L, et al. Prenatal exposure to antibiotics, cesarean section and risk of childhood obesity. Int J Obes (Lond) 2015;39:665-70.

7. Miller SA, Wu RKS, Oremus M. The association between antibiotic use in infancy and childhood overweight or obesity: a systematic review and meta-analysis. Obes Rev 2018 July 23 [Epub ahead of print]. doi: 10.1111/obr.12717.

8. Forbes JD, Azad MB, Vehling L, et al.; Canadian Healthy Infant Longitudinal Development (CHILD) Study Investigators. Association of exposure to formula in the hospital and subsequent infant feeding practices with gut microbiota and risk of overweight in the first year of life. JAMA Pediatr 2018;172:e181161.

9. Medrano-Félix A, Martínez C, Castro-del Campo N, et al. Impact of prescribed cleaning and disinfectant use on microbial contamination in the home. $J$ Appl Microbiol 2011;110:463-71.

10. Schmidt B, Mulder IE, Musk CC, et al. Establishment of normal gut microbiota is compromised under excessive hygiene conditions. PLoS One 2011;6:e28284.

11. Dodson RE, Nishioka M, Standley LJ, et al. Endocrine disruptors and asthmaassociated chemicals in consumer products. Environ Health Perspect 2012;120:935-43.

12. Braun JM. Early-life exposure to EDCs: role in childhood obesity and neurodevelopment. Nat Rev Endocrinol 2017;13:161-73.

13. Bokulich NA, Chung J, Battaglia T, et al. Antibiotics, birth mode, and diet shape microbiome maturation during early life. Sci Transl Med 2016;8:343ra82.

14. Scheepers LE, Penders J, Mbakwa CA, et al. The intestinal microbiota composition and weight development in children: the KOALA Birth Cohort Study. Int J Obes (Lond) 2015;39:16-25.

\section{Competing interests: None declared.}

This article was solicited and has not been peer reviewed.

Affiliations: Department of Epidemiology (Differding, Mueller), and Welch Center for Prevention, Epidemiology and Clinical Research (Mueller), Johns Hopkins Bloomberg School of Public Health, Baltimore, Md.

Contributors: Both authors contributed to the conception and design of the work, drafted the manuscript, revised it critically for important intellectual content, gave final approval of the version to be published and agreed to be accountable for all aspects of the work.

Funding: This article was supported by the Mid-Atlantic Nutrition Obesity Research Center (NORC) under NIH award no. P30DK072488 and the National Heart, Lung, and Blood Institute of the National Institutes of Health under Award Number K01HL141589 (PI: Mueller). The content is solely the responsibility of the authors and does not necessarily represent the official views of the National Institutes of Health.

Correspondence to: Noel Mueller, nmuelle4@jhu.edu 